\title{
OPTIMUM RULES FOR CLASSIFICATION INTO \\ TWO MULTIVARIATE NORMAL POPULATIONS \\ WITH THE SAME COVARIANCE MATRIX
}

\author{
by \\ Somesh Das Gupta * \\ University of Minnesota \\ Technical Report No. 359 \\ October 1979
}

* Supported by a grant from the Mathematics Division, U.S. Army Research Office, Durham, N.C. Grant No. DAAG-29-0038. 


\section{Introduction.}

Let $w$ denote an experimental unit drawn randomly from a population $\pi$. The classification problem in its standard form is to devise rules so as to identify $\pi$ with one of the two given "distinct" populations $\pi_{1}$ and $\pi_{2}$. A set of $p$ real-valued measurements $\underset{\sim}{X}: p \times 1$ is observed on $w$ and it is believed that the distributions of $X$ in those two populations are different. In this paper we shall assume that $\underset{\sim}{\mathrm{X}} \sim \mathrm{N}_{\mathrm{p}}(\underset{\sim}{\mu}, \Sigma)$.

Let $\underset{\sim}{\mu}$ denote the mean of $\underset{\sim}{X}$ in the population $\pi_{i} \quad(i=1,2)$, where $\underset{\sim 1}{\mu} \neq{\underset{\sim}{2}}_{2}$. The classification problem is to find "good" rules for deciding whether $\mu=\mu_{1}$ or $\mu=\mu_{2}$. When all the parameters $\mu_{1}$, $\mu_{2}$ and $\Sigma$ are known Wald's decision theory [D] may be used to derive the miminal complete class of decision rules for zero-one loss function. It is given by the following, except for sets of measure zero [2]: The rule $\phi^{k}$ decides $\mu=\mu_{1}$ iff

$$
\left(x-\mu_{1}\right)-\Sigma^{-1}\left(x-\mu_{1}\right)-\left(x-\mu_{2}\right) \cap \Sigma^{-1}\left(x-\mu_{2}\right) \leq k
$$

It can be proved [2] that the rule $\phi^{\circ}$ is the only admissible minimax rule.

However, in practice all the parameters are not known, and in order to differentiate the two populations random (training) samples from both the populations are obtained. It may be remarked that if either of $\mu_{1}$ and $\mu_{2}$ is known it is not necessary to draw samples from both the populations. 
Let $\theta$ stand for $\left(\mu, \mu_{1}, \mu_{2}, \Sigma\right)$, and

$$
\begin{aligned}
& \theta_{1}=\left\{\theta: \mu=\mu_{1},\left(\mu_{1}, \mu_{2}, \Sigma\right) \in \Omega\right\}, \\
& \theta_{2}=\left\{\theta: \mu=\mu_{2},\left(\mu_{1}, \mu_{2}, \Sigma\right) \in \Omega\right\},
\end{aligned}
$$

where $\Omega$ is a known set in the space of $\mu_{1}, \mu_{2}$ and $\Sigma$. It may be noted that in order to control (arbitrarily) both probabilities of incorrect classification certain conditions must be imposed on $\Omega$ and sequential sampling schemes may have to be used [5]. However, in standard practice $\Omega$ is taken to be the set

$$
\Omega=\left\{\left(\mu_{1}, \mu_{2}, \Sigma\right): \mu_{1}, \mu_{2}, \in R^{P}, \mu_{1} \neq \mu_{2}, \Sigma\right. \text { is positive- }
$$
definite\}.

Following Fisher [7] a set of heuristic rules (called plug-in rules) may be devised by first choosing some good estimates of the unknown parameters and replacing the unknown paramaters in $\phi^{k}$ by their respective estimates. We shall call such a rule $\phi_{p}^{k}$ when the standard estimates are used.

Let $x_{i l}, \ldots, x_{i n}$ denote the $x$-oberservations of the training sample from $\pi_{i}(i=1,2)$. Define (assume $\eta_{1}+n_{2}-2>0$ ) (1..5) $\quad \bar{x}_{i}=\sum_{n_{1}^{j}=1}^{n_{1}} x_{i j} / n_{i} \quad(i=1,2)$, $S=\left[\sum_{j=1}^{1}\left(x_{i j}-\bar{x}_{1}\right)\left(x_{i j}-\bar{x}_{1}\right)^{\prime}+\sum_{j=1}^{2}\left(x_{2 j}-\bar{x}_{2}\right)\left(x_{i j}-\bar{x}_{2}\right)^{\prime}\right] /\left(n_{1}+n_{2}-2\right)$ 
When all the parameters are unknown, Fisher's plug-in rules are given by the following: The rule $\phi_{p}^{k}$ decides $\mu=\mu_{1}$ iff (1.6) $\quad\left(x-\bar{x}_{1}\right)-s^{-1}\left(x-\bar{x}_{1}\right)-\left(x-\bar{x}_{2}\right)-s^{-1}\left(x-\bar{x}_{2}\right) \leq k$

Using the likelihood-ratio principle Anderson [1] proposed the following rules when $\left(\mu_{1}, \mu_{2}, \Sigma\right)$ lies in $\Omega$ given by $(1.4)$ : The rule $\psi_{L}^{\lambda}$ decides $\mu=\mu_{1}$ iff

$$
\begin{aligned}
& \left(1+1 / n_{1}\right)^{-1}\left(x-\bar{x}_{1}\right)^{-} s^{-1}\left(x-\bar{x}_{1}\right)-\lambda\left(1+1 / n_{2}\right)^{-1} \\
& \left(x-\bar{x}_{2}\right)^{-} s^{-1}\left(x-x_{2}\right) \leq \lambda-1,
\end{aligned}
$$

where $S^{*}=\mathrm{mS}, \mathrm{m}=\mathrm{n}_{1}+\mathrm{n}_{2}-2 . \quad(\lambda>0)$ Note that $\Psi_{L}^{1}=\phi_{\mathrm{p}}^{0}$ when $n_{1}=n_{2}$. The likelihood-ratio rules turn out to be the following when $\Sigma$ is known: The rule $\phi_{L}^{k}$ decides $\mu=\mu_{i}$ iff

$$
\begin{aligned}
& \left(1+1 / \mathrm{n}_{1}\right)^{-1}\left(\mathrm{x}-\overline{\mathrm{x}}_{1}\right)^{-} \Sigma^{-1}\left(\mathrm{x}-\overline{\mathrm{x}}_{1}\right) \\
& \quad-\left(1+1 / \mathrm{n}_{2}\right)^{-1}\left(\mathrm{x}-\overline{\mathrm{x}}_{2}\right)^{-1} \Sigma^{-1}\left(\mathrm{x}-\overline{\mathrm{x}}_{2}\right) \leq \mathrm{k}
\end{aligned}
$$

One may also derive some "good" constructive rules from various optimality criteria. In this paper we shall obtain some good rules from Wald's decision-theoretic viewpoint, and also from asymmetrical Neyman-Pearson approach. We shall also study the above two classes of heuristic rules from some optimality criteria.

\section{The Univariate Case}

2-1. $p=1, \sigma^{2}$ is known. Without any loss of generality we shall assume that $\sigma^{2}=1$. Let $\varphi=\left(\varphi_{1}, \varphi_{2}\right)$ stand for a decision rule, where $\varphi_{i}$ 
is the probability of deciding $\mu=\mu_{i}$ given the observations. We shall consider only the rules based on sufficient statistics $x, \bar{X}_{1}$ and $\bar{X}_{2}$.

First we shall make an orthogonal transformation as follows: Define

$$
\begin{aligned}
& \mathrm{u}_{1}=\mathrm{k}_{1}\left[\left(1+1 / \mathrm{n}_{1}\right)^{-\frac{1}{2}}\left(\mathrm{x}-\overline{\mathrm{X}}_{1}\right)+\left(1+1 / \mathrm{n}_{2}\right)^{-\frac{1}{2}}\left(\mathrm{x}-\overline{\mathrm{x}}_{2}\right)\right], \\
& \mathrm{u}_{2}=\mathrm{k}_{2}\left[\left(1+1 / \mathrm{n}_{1}\right)^{-\frac{1}{2}}\left(\mathrm{x}-\overline{\mathrm{x}}_{1}\right)-\left(1+1 / \mathrm{n}_{2}\right)^{-\frac{1}{2}}\left(\mathrm{x}-\overline{\mathrm{x}}_{2}\right)\right], \\
& \mathrm{U}_{3}=\mathrm{k}_{3}\left[\mathrm{X}+\mathrm{n}_{1} \overline{\mathrm{x}}_{1}+\mathrm{n}_{2} \overline{\mathrm{x}}_{2}\right],
\end{aligned}
$$

where $k_{i}$ 's are chosen so that $\operatorname{var}\left(U_{i}\right)=1 ; i=1,2,3$. Note that $U_{i}$ 's are independently distributed. Let $E\left(U_{i}\right)=\nu_{i}$. Then $U_{i} \sim N\left(\nu_{i}, 1\right)$.

In terms of $\left(\nu_{1}, \nu_{2}, \nu_{3}\right)$ the sets $\theta_{1}$ and $\theta_{2}$ as defined in $(1.2)-(1.4)$, are transformed as follows:

$$
\begin{aligned}
& \Omega_{1}=\left\{\left(\nu_{1}, \nu_{2}, \nu_{3}\right): \nu_{1}=\nu, \nu_{2}=-c \nu, \nu \neq 0, \nu_{3} \in R\right\} \\
& \Omega_{2}=\left\{\left(\nu_{1}, \nu_{2}, \nu_{3}\right): \nu_{1}=\nu, \nu_{2}=c \nu, \nu \neq 0, \nu_{3} \in R\right\},
\end{aligned}
$$

where $c=k_{2} / k_{1}>0$. ( $k_{i}$ 's are chosen to be positive.) Note that $c>1$.

\subsubsection{Bayes Rules and Minimax Rules}

It is easy to see that by taking a suitable prior distribution of $\nu_{3}$ independently of $\nu_{1}$ and $\nu_{2}$ we can get Bayes rules free from $U_{3}$. Hence we shall only consider prior distributions of $\left(\nu_{1}, \nu_{2}\right)$ and drop $U_{3}$ from the argument of $\varphi$. Let

$$
\Omega_{i}^{*}=\left\{\left(\nu_{1}, \nu_{2}\right): \nu_{1}=\nu, \nu_{2}=(-1)^{i} c \nu, \nu \neq 0\right\}
$$

Consider a prior distribution $\xi\left(\beta, \gamma, \nu_{0}\right)$ which assigns probabilities $\beta \gamma,(1-\beta)(1-\gamma), \beta(1-\gamma), \gamma(1-\beta)$ to the parameter points $\left(\nu_{0}, c \nu_{0}\right),\left(-\nu_{0},-c \nu_{0}\right)$, $\left(\nu_{0},-c \nu_{0}\right),\left(-\nu_{0}, c \nu_{0}\right)$, respectively, where $0 \leq \beta \leq 1,0 \leq \gamma \leq 1, \nu_{0}>0$. It can be seen that the unique (a.e) Bayes rule (for zero-one loss function) against the above prior distribution is given by the following: Decide $\left(\nu_{1}, \nu_{2}, \nu_{3}\right) \in \Omega_{1}$ iff 


$$
\left(U_{1}-c_{1}\right)\left(U_{2}-c_{2}\right) \leq 0
$$

where $c_{1}$ and $c_{2}$ are functions of $\nu_{0}, \beta, \gamma$ and $c$. Conversely, given $c_{1}$ and $c_{2}$ it is possible to choose $\beta, \gamma$ and $\nu_{0}$ appropriately. Another class of Bayes rules may be obtained from the following prior distributions: The probability that $\left(\nu_{1}, \nu_{2}\right) \in \Omega_{i}^{*}$ is $\xi_{i}$, and given that $\nu_{1}=\nu$, $v_{2}=(-1)^{i} c \nu$ the distribution of $\nu$ is $N\left(0, \tau^{2}\right)$. The unique (a.e) Bayes rule against the above prior distribution decides $\left(\nu_{1}, \nu_{2}, \nu_{3}\right) \in \Omega_{1}$ iff (2.8) $\quad \mathrm{U}_{1} \mathrm{U}_{2} \leq \mathrm{k}$, where $k$ is a function of $\xi_{1}, \xi_{2}$ and $c$. Different types of Bayes rules are given by DasGupta and Bhattacharya [ 3 ].

Now consider the rule which decides $\left(\nu_{1}, \nu_{2}, \nu_{3}\right) \in \Omega_{1}$ iff

$$
\mathrm{U}_{1} \mathrm{U}_{2} \leq 0
$$

Note that $(2.9)$ is equivalent to

$$
\left(1+1 / n_{1}\right)^{-1}\left(x-\bar{x}_{1}\right)^{2} \leq\left(1+1 / n_{2}\right)^{-1}\left(x-\bar{x}_{2}\right)^{2} \text {. }
$$

Thus the above rule is the same as $\varphi_{L}^{0}$, defined in (1.8). The rule $\varphi_{\mathrm{L}}^{0}$ is the unique Bayes rule against the prior $\xi\left(\frac{1}{2}, \frac{1}{2}, v_{0}\right)$ for any $\nu_{0}>0$. Moreover, the risk of the rule $\varphi_{L}^{0}$ is constant over the four-point set $\left(\nu_{0}, c \nu_{0}\right),\left(-\nu_{0},-c \nu_{0}\right),\left(-\nu_{0}, c \nu_{0}\right),\left(\nu_{0},-c \nu_{0}\right)$. Hence $\varphi_{L}^{0}$ is an admissible minimax rule, and moreover the supremum of the risk of $\varphi_{\mathrm{L}}^{0}$ is equal to $\frac{1}{2}$. However, $\varphi_{\mathrm{L}}^{0}$ is not the unique minimax rule (leaving aside the trivial rule $\left.\varphi_{1} \equiv \varphi_{2} \equiv \frac{1}{2}\right)$. To see this, transform $\left(U_{1}, \mathrm{U}_{2}\right)$ to $\left(V_{1}, V_{2}\right)$ by an orthogonal transformation $L$ such that $\left(E \cdot V_{1}, E \cdot v_{2}\right)$ is proportional to $\left(1,-d_{1}\right)$ and $\left(1, d_{2}\right)$ for $\left(\nu_{1}, \nu_{2}\right) \in \Omega_{1}^{*}$ and $\left(\nu_{1}, \nu_{2}\right) \in \Omega_{2}^{*}$, respectively, and $d_{1}>0$, $\mathrm{d}_{2}>0$. Let $\psi$ be the rule which decides $\left(\nu_{1}, \nu_{2}\right) \in \Omega_{1}^{*}$ iff $v_{1} v_{2} \leq 0$. It can be easily seen (or, see [6]) that the supremum of the risk of $\psi$ is $\frac{1}{2}$. Note that there are many such orthogonal transformations $L$ which will 
satisfy the desired property for $\left(E v_{1}, E v_{2}\right)$. It may be shown that neither of the rules $\varphi_{L}^{0}$ and $\psi$ dominates the other. However, the characterization of the class of all admissible minimax rules is not known.

Now, instead of the zero-one loss function consider a loss function which takes the value 0 for correct decisions and equals $\ell\left(\left|\mu_{1}-\mu_{2}\right|\right)$ for any incorrect decision, where $l$ is a positive-valued bounded, continuous function such that $\ell(\Delta) \rightarrow 0$ as $\Delta \downarrow 0$. DasGupta and Bhattacharya [3] have shown that $\varphi_{\mathrm{L}}^{0}$ is the unique minimax rule (and Bayes admissible) for the above loss function when $n_{1}=n_{2}$.

It is clear that neither of $\varphi_{p}^{0}$ and $\varphi_{\mathrm{L}}^{0}$ dominates the other. It is believed that $\varphi_{\mathrm{p}}^{0}$ is also admissible.

\subsubsection{Invariant Rules. Let us now consider the following conditions on} the rules based on $\mathrm{U}_{1}, \mathrm{U}_{2}, \mathrm{U}_{3}$ :

Translation invariance:

$$
\varphi\left(u_{1}, u_{2}, u_{3}\right)=\varphi\left(u_{1}, u_{2}, u_{3}+b\right)
$$

for all $u_{1}, u_{2}, u_{3}$ and $b \in R$.

A set of maximal invariants for $(2.11)$ is given by $\left(U_{1}, U_{2}\right)$. Hence we shall write a translation-invariant rule as a function of $U_{1}$ and $U_{2}$.

\section{Sign invariance:}

$$
\varphi\left(u_{1}, u_{2}, u_{3}\right)=\varphi\left(-u_{1},-u_{2},-u_{3}\right)
$$

for all $u_{1}, u_{2}, u_{3}$.

A translation-invariant rule is sign-invariant iff it is a function of $\left(u_{1} u_{2} /\left|u_{2}\right|,\left|u_{2}\right|\right) \cdot[10]$.

Symmetry:

$$
\varphi_{1}\left(u_{1},-u_{2}, u_{3}\right)=\varphi_{2}\left(u_{1}, u_{2}, u_{3}\right)
$$

for all $u_{1}, u_{2}$ and $u_{3}$. 
It is clear that both $\Omega_{1}$ and $\Omega_{2}$ are unchanged under the transformations $\left(u_{1}, u_{2}, u_{3}\right) \rightarrow\left(u_{1}, u_{2}, u_{3}+c\right)$ and $\left(u_{1}, u_{2}, u_{3}\right) \rightarrow\left(-u_{1},-u_{2},-u_{3}\right)$. In terms of $x, \bar{x}_{1}$ and $\bar{x}_{2}$ these transformations are respectively $\left(x, \bar{x}_{1}, \bar{x}_{2}\right) \rightarrow\left(x+b, \bar{x}_{1}+b, \bar{x}_{2}+b\right)$ and $\left(x, \bar{x}_{1}, \bar{x}_{2}\right) \rightarrow\left(-x,-\bar{x}_{1},-\bar{x}_{2}\right)$.

The sets $\Omega_{1}$ and $\Omega_{2}$ are interchanged under the transformation $\left(u_{1}, u_{2}, u_{3}\right) \rightarrow\left(u_{1},-u_{2}, u_{3}\right)$. This transformation is obtained by interchanging $\left(\bar{x}_{1}, n_{1}\right)$ and $\left(\bar{x}_{2}, n_{2}\right)$. We shall now show that $\varphi_{L}^{0}$ is the uniformly best translation-invariant, sign-invariant symetric rule. For $\left(\nu_{1}, \nu_{2}, \nu_{3}\right) \in \Omega_{1}$ (i.e., $\nu_{1}=\nu, \nu_{2}=-c \nu$ ) the risk of a translation-invariant, sign-invariant symetric rule $\varphi$ is given by

$$
\begin{aligned}
& E_{\nu_{1}, v_{2}, \nu_{3}} \varphi_{2}\left(u_{1}, u_{2}\right) \\
& =\int_{0}^{\infty} \int_{0}^{\infty}\left[\varphi_{2}\left(u_{1}, u_{2}\right) n\left(u_{1} ; \nu\right) n\left(u_{2} ;-c \nu\right)\right. \\
& +\varphi_{2}\left(u_{1}, u_{2}\right) n\left(u_{1} ;-\nu\right) n\left(u_{2}, c \nu\right) \\
& +\left\{1-\varphi_{2}\left(u_{1}, u_{2}\right)\right\}_{n}\left(u_{1} ;-v\right) n\left(u_{2} ;-c \nu\right) \\
& +
\end{aligned}
$$

where $n(u ; \nu)$ is the density of $N(\nu, 1)$ at $u$. It may be seen that (2.14) is minimum (uniformly in $v$ and $\left.v_{3}\right)$ for $\varphi_{2}\left(u_{1}, u_{2}\right)=1$ when $u_{1} u_{2}>0$. The above result can also be proved using the distribution of $\left(U_{1} U_{2} /\left|U_{2}\right|,\left|U_{2}\right|\right)$ [10].

Kinderman [10] characterized the (essential) complete class among all translation-invariant, sign-invariant rules when $\mathrm{n}_{1}=\mathrm{n}_{2}$.

2.1.3 Best Invariant Similar Test. The classification problem may be viewed in the light of Neyman-Pearson Theory. We may pose the problem as testing the hypothesis $\mathrm{H}_{1}: \theta \in \theta_{1}$ against the alternative $\theta \in \theta_{2}$. We restrict our attention to the class of tests which are translation-invariant and 
sign-invariant. Let $\psi$ be a test function, i.e. $\psi\left(x, \bar{x}_{1}, \bar{x}_{2}\right)$ is the probability of rejecting $\mathrm{H}_{1}$ given $\mathrm{x}, \overline{\mathrm{x}}_{1}$ and $\overline{\mathrm{x}}_{2}$. Define

(2.15) $\quad Y_{1}=\left(1+1 / n_{1}\right)^{-\frac{1}{2}}\left(x-\bar{X}_{1}\right)$,

(2.16) $\quad Y_{2}=\left[\left(1+1 / \mathrm{n}_{2}\right)^{-\frac{1}{2}}\left(\mathrm{X}-\overline{\mathrm{X}}_{2}\right)-\left(1+1 / \mathrm{n}_{2}\right)^{-\frac{1}{2}}\left(1+1 / \mathrm{n}_{1}\right)^{-1}\left(\mathrm{X}-\overline{\mathrm{X}}_{1}\right)\right] \mathrm{d}$,

(2.17) $\quad Y_{3}=\left(1+n_{1}+n_{2}\right)^{-\frac{1}{2}}\left(x+n_{1} \bar{X}_{1}+n_{2} \bar{X}_{2}\right)$,

where $d$ is a constant chosen appropriately to make $\operatorname{var}\left(\mathrm{Y}_{2}\right)=1$. If $\psi$ is translation-invariant it will depend only on $\mathrm{Y}_{1}$ and $\mathrm{Y}_{2}$. Furthermore the sign-invariance of $\psi$ means

$$
\psi\left(y_{1}, y_{2}\right)=\psi\left(-y_{1},-y_{2}\right)
$$

Under $H_{1}$ the means of $Y_{1}$ and $Y_{2}$ are given by

$$
\delta_{1} \equiv E Y_{1}=0, \quad \delta_{2}=E Y_{2}=d\left(1+1 / n_{2}\right)^{-\frac{3}{2}}\left(\mu_{1}-\mu_{2}\right) \text {. }
$$

Similarly, the means of $\mathrm{Y}_{1}$ and $\mathrm{Y}_{2}$ under $\mathrm{H}_{2}$ are given by

$$
\delta_{1}=\left(1+1 / n_{1}\right)^{-\frac{1}{2}}\left(\mu_{2}-\mu_{1}\right), \delta_{2}=-d\left(1+1 / n_{2}\right)^{-\frac{1}{2}}\left(1+1 / n_{1}\right)^{-1}\left(\mu_{2}-\mu_{1}\right) \text {. }
$$

In terms of $\delta_{1}$ and $\delta_{2}$ the parameter sets may be expressed as

$$
\Delta_{1}=\left\{\left(\delta_{1}, \delta_{2}\right): \delta_{1}=0, \delta_{2} \neq 0\right\} \text {, }
$$

$$
\Delta_{2}=\left\{\left(\delta_{1}, \delta_{2}\right): \delta_{2}=a \delta_{1} \neq 0\right\}
$$

under $\mathrm{H}_{1}$ and $\mathrm{H}_{2}$, respectively; $a=-d\left(1+1 / \mathrm{n}_{1}\right)^{-\frac{1}{2}}\left(1+1 / \mathrm{n}_{2}\right)^{-\frac{1}{2}}$. Since $\delta_{2}$ is still unknown under $\mathrm{H}_{1}$ we require $\psi$ to be similar size $\alpha$ for $\mathrm{H}_{1}$, i.e.

$$
E_{0, \delta_{2}} \psi\left(Y_{1}, Y_{2}\right)=\alpha \text { for all } \delta_{2} \neq 0 \text {. }
$$

This is equivalent to

$$
\text { (2.24) } \int_{-\infty}^{\infty} \psi\left(y_{1}, y_{2}\right) n\left(y_{1} ; 0\right) d y_{1}=\alpha \quad \text { a.e. }\left(y_{2}\right) \text {. }
$$

The power of the test $\psi$ is given by 


$$
\begin{aligned}
& \mathrm{E}_{\delta_{1}, \delta_{2}} \psi\left(\mathrm{Y}_{1}, \mathrm{Y}_{2}\right)=\frac{1}{2}\left[\mathrm{E}_{\delta_{1}, \delta_{2}} \psi\left(\mathrm{Y}_{1}, \mathrm{Y}_{2}\right)+\mathrm{E}_{-\delta_{1},-\delta_{2}} \psi\left(\mathrm{Y}_{1}, \mathrm{Y}_{2}\right)\right] \\
& =\frac{1}{2} \int_{-\infty-\infty}^{\infty} \int_{-\infty}^{\infty} \mathrm{e}^{-\delta_{1}^{2}\left(1+\mathrm{a}^{2}\right) / 2} \mathrm{n}\left(\mathrm{y}_{1} ; 0\right) \mathrm{n}\left(\mathrm{y}_{2} ; 0\right) \psi\left(\mathrm{y}_{1}, \mathrm{y}_{2}\right) \\
& {\left[e^{\delta_{1}\left(y_{1}+a y_{2}\right)}+e^{-\delta_{1}\left(y_{1}+a y_{2}\right)}\right] d y_{1} d y_{2} .}
\end{aligned}
$$

Using the Neyman-Pearson Lemma in order to maximize

$$
\int_{-N}^{\infty} \psi\left(y_{1}, y_{2}\right)\left[e^{\delta_{1}\left(y_{1}+a y_{2}\right)}+e^{-\delta_{1}\left(y_{1}+a y_{2}\right)}\right] n\left(y_{1}, 0\right) d y_{1} .
$$

subject to (2.24) we get the following optimum test:

$$
\psi^{*}\left(y_{1}, y_{2}\right)=1 \text { iff }\left|y_{1}+a y_{2}\right|>k\left(y_{2}\right)
$$

where $k\left(y_{2}\right)$ is chosen so that

$$
\int_{-a y_{2}-k\left(y_{2}\right)}^{-a y_{2}+k\left(y_{2}\right)} n\left(y_{1} ; 0\right) d y_{1}=I-\alpha \text {. }
$$

Thus $\psi^{*}$ is the uniformly most powerful invariant similar test. The above result is due to Schaafsma [12].

\subsection{The common variance $\sigma^{2}$ is unknown}

It may be easily seen that the rules given by (2.7) and (2.8) are still unique Bayes. Moreover, the rule $\psi_{L}^{I}$ is the one which accepts $\theta \in \theta_{1}$ if. (2.10) holds and it is admissible minimax. When $n_{1}=n_{2}$ Das Gupta and Bhattacharya [3] have shown that the rule $\psi_{L}^{1}$ is the unique (a.e.) mini$\max$ when the loss for incorrect decision is $\ell\left(\left|\mu_{1}-\mu_{2}\right| / \sigma\right)$, where $\ell$ is a positive valued, bounded, continuous function such that $\ell(\Delta) \rightarrow 0$ as $\Delta \downarrow 0$. To see all the above results, not that $\left(U_{1}, U_{2}, U_{3}, S\right)$ are sufficient statistics in this case and $S$ is distributed independently of $\left(\mathrm{U}_{1}, \mathrm{U}_{2}, \mathrm{U}_{3}\right)$. It also follows that $\psi_{L}^{I}$ is the uniformly best translation-invariant, symmetric rule. To see this, condition on $S$ and fix $\sigma$,

Schaafsma [13] has shown that the following critical region for testing $\mathrm{H}_{1}$ against $\mathrm{H}_{2}$ is (i) similar of size $\alpha$ for $\mathrm{H}_{1}$, (ii) unbiased for $\mathrm{H}_{2}$, and 
(iii) asymptotically (as $\min \left(\mathrm{n}_{1}, \mathrm{n}_{2}\right) \rightarrow \infty$ ) most stringent among all level

$\alpha$ tests:

$$
Y_{1} \operatorname{sign}\left(Y_{2}\right)>\sqrt{S} t_{n_{1}}+n_{2}-2, \alpha,
$$

where $Y_{1}$ and $Y_{2}$ are given in (2.15) and (2.16), $S$ is given in (1.5), and $t_{n_{1}}+n_{2}-2, \alpha$ is the upper $100 \alpha \%$ point of the Student's $t$ distribution with $n_{1}+n_{2}-2$ degrees of freedom. However, it is very likely that this test is not admissible.

It follows from Kiefer and Schwartz [9] that the rule $\psi_{L}^{\lambda}$ is a (unique) Bayes rule. We shall give a sketch of the prior distribution against which $\psi_{\mathrm{L}}^{\lambda}$ is unique Bayes. Consider $\mathrm{U}_{1}, \mathrm{U}_{2}, \mathrm{U}_{3}$ as defined in (2.1) - (2.3). Then $U_{i}^{\prime}$ 's are independently distributed, and $U_{1} \sim N\left(\nu_{i}, \sigma^{2}\right)$. Moreover, under $\theta \in \Theta_{i}$ (i.e. $\left(\nu_{1}, \nu_{2}, \nu_{3}\right) \in \Omega_{i}$ ) we have $\nu_{1}=\nu, \nu_{2}=(-1)^{i} c \nu, \nu \neq 0$. The prior distribution is given as follows:

(i) $P\left(\theta \in \Theta_{i}\right)=\xi_{i}, \quad i=1,2$.

(ii) Given $\theta \in \theta_{i}$, the conditional distribution of $\left(\nu, \nu_{3}, \sigma^{2}\right)$ is derived from the following:

(iia) Given $\sigma^{2}=\left(1+\tau^{2}\right)^{-1}$, the conditional distribution of $\left(\frac{\nu}{\sigma^{2}}, \frac{\nu_{3}}{\sigma^{2}}\right)$ is the same as that of $\left(\tau V, \tau \cdot V_{3}\right)$, where $V$ and $v_{3}$ are independently distributed with $V \sim N\left(0,\left(1+\tau^{2}\right) /\left(1+c^{2}\right)\right)$ and $V_{3} \sim N\left(0,1+\tau^{2}\right)$.

(iib) The density of $\tau$ is proportional to $\left(1+\tau^{2}\right)^{-(m+I) / 2}$. 


\section{Multivariate Case: $\sum$ known}

Without any loss of generality we shall assume that $\Sigma=I_{p}$. First we shall derive a class of Bayes rules and obtain an admissible minimax rule. Define $\mathrm{U}_{1}, \mathrm{U}_{2}, \mathrm{U}_{3}$ and $\mathrm{k}_{1}, \mathrm{k}_{2}$ as in (2.]) - (2.3), except that $U_{i}^{\prime}$ 's are now $p \times 1$ vectors and $U_{i} \sim \mathbb{N}_{p}\left(\nu, I_{p}\right)$. Correspondingly redefine the sets $\Omega_{i}$ as follows:

$$
\Omega_{i}=\left\{\left(\nu_{1}, \nu_{2}, \nu_{3}\right): \quad \nu_{1}=\nu, \nu_{2}=(-1)^{i} c \nu, \neq 0 ; \nu, \nu_{3} \in R^{p}\right\} \text {, }
$$

$i=1,2$. As before $U_{3}$ may be eliminated from a Bayes rule by taking a fixed distribution, independent of $\left(\nu_{1}, \nu_{2}\right)$, under both $\Omega_{1}$ and $\Omega_{2}$. Now consider the prior distribution which assigns the probability $\xi_{i}$ to $\Omega_{i}$ and, given $\nu_{1}=\nu, \nu_{2}=(-1)^{1} c \nu$, the distribution of $\nu$

is $N_{p}\left(0, \tau^{2} I_{p}\right)$. It can now be seen that the unique (a.e.) Bayes rule against the above prior distribution decides $\left(\nu_{1}, \nu_{2}, \nu_{3}\right) \in \Omega_{1}$ iff

$$
\mathrm{u}_{1}^{\sim} \mathrm{U}_{2} \leq \mathrm{k}
$$

where $k$ is a function of $\xi_{1}$ and $\xi_{2}$; conversely, given $k$ the probability $\xi_{1}$ and $\xi_{2}$ can be suitably chosen. Thus any likelihoodratio rule $\varphi_{L}^{k}$ is Bayes and admissible.

We shall now show that $\varphi_{L}^{0}$ is minimax. First we shall consider a different prior distribution against which $\varphi_{L}^{0}$ is unique Bayes. As before, $\nu_{3}$ can be eliminated from the problem. Now consider a prior distribution which assigns equal probabilities to the sets $\Omega_{1}^{*}$ and $\Omega_{2}^{*}$, where

$$
\Omega^{*}=\left\{\left(\nu_{1}, \nu_{2}\right): \nu_{1}=\nu, \nu_{2}=(-1)^{i} c \nu, \nu \neq 0, \nu \in R^{p}\right\} .
$$


Moreover, given that $\left(\nu_{1}, \nu_{2}\right) \in \Omega_{i}^{*}$, the distribution of $\nu$ is taken to be uniform over the surface of the hypershpere $v^{\prime} \nu=\Delta^{2}$. See Das Gupta [4] to get a detailed proof of the fact that $\phi_{L}^{0}$ is unique (a.e.) Bayes against the above prior distribution. To see that $\phi_{L}^{0}$ is minimax, note that the risk of $\phi_{I}^{0}$ is constant over the set

$$
\begin{aligned}
& \left\{\left(\nu_{1}, \nu_{2}, \nu_{3}\right): \nu_{1}=\nu, \nu_{2}=-c \nu, \nu^{\prime} \nu=\Delta^{2}\right\} . \\
& \text { U }\left\{\left(\nu_{1}, \nu_{2}, \nu_{3}\right): \quad \nu_{1}=\nu, \nu_{2}=c \nu, \nu^{\prime} \nu=\Delta^{2}\right\} .
\end{aligned}
$$

Das Gupta [4] has also shown that the rule $\phi_{L}^{0}$ is the unique (a.e.) minimax when the loss for any correct decision is zero, and the loss for deciding $\mu=\mu_{i}$ incorrectly is

$$
\ell\left[\left(1+1 / n_{i}\right)^{-1}\left(\mu-\mu_{i}\right)^{-}\left(\mu-\mu_{i}\right)\right]
$$

where $\ell$ is a positive-valued, bounded, continuous function such that $\ell(\Delta) \rightarrow 0$ as $\Delta+0$.

As in (2.11) we may call a rule $\phi$ translation-invariant if

$$
\phi\left(\mathrm{U}_{1}, \mathrm{U}_{2}, \mathrm{U}_{3}\right)=\phi\left(\mathrm{U}_{1}, \mathrm{U}_{2}, \mathrm{U}_{3}+\mathrm{b}\right)
$$

for all $b \in R^{p}$. Clear̂ly, $\left(U_{1}, U_{2}\right)$ is a set of maximal invariants. A rule $\phi$ is called orthogonally-invariant if

$$
\phi\left(U_{1}, U_{2}, U_{3}\right)=\phi\left(O U_{1}, O U_{2}, O U_{3}\right)
$$

for all orthogonal $p \times p$ matrices 0 .

Kudo [11] considered the following "symmetry" condition for a translation-invariant rule $\phi$ :

$$
\beta_{1}\left(\phi ;\left(1+1 / n_{2}\right)^{-1 / 2} d\right)=B_{2}\left(\phi ;\left(1+1 / n_{1}\right)^{-1 / 2} d\right),
$$

where $\beta_{i}(\phi ; d)=E_{\theta} \phi_{i}$ when $d=\left(\mu_{1}-\mu_{2}\right)$. and $\mu=\mu_{i}$. Moreover, he 
required $B_{i}(\phi ; d)$ to depend on $d$ only through $d^{-} d$. This condition clearly holds if $\phi$ is translation-invariant and orthogonallý-invariant. Note also that for a translation-invariant and an orthogonally-invariant rule $\phi$ satisfying (2.13) the condition (3.9) holds. Kudo [11] has shown that $\phi_{L}^{0}$ simultaneously maximizes both $\beta_{1}(\phi ; d)$ and $B_{2}(\phi ; d)$ in the class of all translation-invariant rules satisfying (3.9) and for which $B_{i}(\phi ; d)$ depends on $d$ only through $d^{\prime} d$. This can be seen easily by integrating the probability of correct classification with respect to the uniform distribution of $v$ over $v^{\prime} v=\Delta^{2}$, where $v_{1}=v, v_{2}=(-1)^{i} \mathrm{c} v$. Rao [15] has considered the class $\Phi^{*}$ of rules whose probabilities of misclassification depend only on

(3.10) $\Delta^{2}=\left(\mu_{1}-\mu_{2}\right) \cdot \Sigma^{-1}\left(\mu_{1}-\mu_{2}\right)$.

For a rule $\phi \in \Phi^{*}$ let $G_{1}\left(\phi ; \Delta^{2}\right)$ and $G_{2}\left(\phi ; \Delta^{2}\right)$ be the error probabilities when $\mu=\mu_{1}$ and $\mu=\mu_{2}$, respectively. Rao [15] has posed the problem of minimizing

$$
\left.\frac{\mathrm{d}}{\mathrm{d} \Delta^{2}}\left\{\mathrm{aG} \mathrm{G}_{1}\left(\phi ; \Delta^{2}\right)+\mathrm{b} \mathrm{G}_{2}\left(\phi: \Delta^{2}\right)\right\}\right|_{\Delta=0},
$$

subject to the condition that the ratio of $G_{1}(\phi ; 0)$ to $G_{2}(\phi ; 0)$ is equal to some specified constant. The resulting optimum rule decides $\mu=\mu_{1}$ iff

$$
\begin{aligned}
& a\left[\left(x-\bar{x}{ }_{1}\right)-\left(1+1 / n_{1}\right)\left(x-\bar{x}_{2}\right)\right]^{-}\left[\left(x-\bar{x}{ }_{1}\right)-\left(1+1 / n_{1}\right)\left(x-\bar{x}_{2}\right)\right] \\
& -b\left[\left(1+1 / n_{2}\right)\left(x-\bar{x}_{1}\right)-\left(x-\bar{x}_{2}\right)\right]^{-}\left[\left(1+1 / n_{2}\right)\left(x-\bar{x}_{1}\right)-\left(x-\bar{x}_{2}\right)\right] \geq k
\end{aligned}
$$

The above rule coincides with $\phi_{L}^{0}$ when $n_{1}=n_{2}$ and $a=b, k=0$. 


\section{Multivariate Case: $\sum$ unknown}

First we shall show that a likelihood-ratio rule $\Psi_{L}^{\lambda}$ is unique (a.e.) Bayes and hence is admissible (for zero-one loss function). Note that $\mathrm{U}_{1}, \mathrm{U}_{2}, \mathrm{U}_{3}$ and $\mathrm{S}$ are sufficient statistics in this case, where $\mathrm{U}_{i}$ 's (in $p \times 1$ vector notations) are given by (2.1) $-(2.3)$ and $S$ is given by (1.5). Here $U_{i} \sim N_{p}\left(\nu_{i}, \Sigma\right)$. We now consider the following prior distribution.

(i) $\underline{p}\left(\theta \in \theta_{i}\right)=\xi_{i}$

(ii) Given $\theta \in \theta_{i}$ (i.e., $\nu_{1}=\nu, \nu_{2}=(-1)^{i} c \nu$ ), the conditional distribution of $\left(\nu, \nu_{3}, \Sigma\right)$ is derived from the following:

(iia) Given $\Sigma^{-1}=I_{p}+\tau \tau^{\prime}(\tau: p \times 1)$, the conditional distribution of $\left(\Sigma^{-1} \nu, \Sigma^{-1} \nu_{3}\right)$ is the same as the distribution of $\left(\tau V, \tau V_{3}\right)$, where $V$ and $V_{3}$ are independently distributed as

$$
N\left(0,\left(1+c^{2}\right)^{-1}\left(1+\tau^{-} \tau\right)\right) \text { and } N\left(0,1+\tau^{-} \tau\right)
$$

respectively.

(iib) The density of $\tau$ is proportional to $\left(1+\tau^{-} \tau\right)^{-(m+1) / 2}$, where $m>p-1$.

Following a simplified version of the results of Kiefer and Schwartz [9] it can be shown that a unique (a.e.) Bayes rule against the above prior distribution accepts $\mu=\mu_{1}$ if (1.7) holds, where $\lambda$ is a function of $\xi_{i}$ 's; conversely, given $\lambda$ the constants $\xi_{i}$ 's can by appropriately chosen.

Das Gupta [4] has considered aclass $\bar{\phi}^{* *}$ of rules invariant under the following transformations:

$(4.1$

$$
\left(\mathrm{x}, \overline{\mathrm{x}}_{1} \overline{\mathrm{x}}_{2}, \mathrm{~S}\right) \rightarrow\left(\mathrm{AX}+\mathrm{b}, \mathrm{A} \overline{\mathrm{x}}_{1}+\mathrm{b}, \mathrm{A} \overline{\mathrm{x}}_{2}+\mathrm{b}, \mathrm{ASA}^{\prime}\right)
$$


where $A$ is any $P \times p$ nonsingular matrix and $b$ is any vector in $R^{P}$. It is shown [ 4 ] that a set of maximal invariants is given by $\left(m_{11}, m_{12}, m_{22}\right)$, where

(4.2) $\quad m_{i j}=U_{i} \cdot s^{-1} U_{j} / m$.

When $v_{1}=v, \nu_{2}=(-1)^{i} c v, v^{-} \Sigma^{-1} v=\Delta^{2}$, the joint density of $\left(m_{11}, m_{12}, m_{22}\right)$ is given by [14]

$$
\mathrm{p}_{i}\left(\mathrm{~m}_{11}, \mathrm{~m}_{12}, \mathrm{~m}_{22} ; \Delta^{2}\right)=\mathrm{K} \exp \left[-\Delta^{2}\left(1+\mathrm{c}^{2}\right) / 2\right]|\mathrm{M}|^{(\mathrm{p}-3) / 2}
$$$$
\sum_{j=0}^{\infty} g_{j}\left(\frac{1}{2} \Delta^{2}\right)^{j} h_{j}\left(m_{11}, m_{12}, m_{22}\right),
$$

where

$$
\text { (4.4) } h_{j}\left(m_{11}, m_{12}, m_{22}\right)=\frac{\left(m_{11}+2(-1)^{i} c_{12}+c^{2} m_{22}+\left(1+c^{2}\right)|M|\right)^{j}}{\left|I_{2}+M\right|^{\frac{1}{2}(m+2)+j}}
$$

(4.5) $|M|=\operatorname{det} M, \quad M=\left(\begin{array}{ll}m_{11} & m_{12} \\ m_{12} & m_{22}\end{array}\right)$,

(4.6) $\quad m=n_{1}+n_{2}-2$,

and $K>0, g_{i}>0$ are numerical constants.

Consider a prior distribution which assign equal probabilities to $\theta_{i}$ and, given $\theta \in \theta_{i}$ (i.e. $\nu_{1}=\nu, \nu_{2}=(-1)^{i} c \nu$ ) the value of $v^{-} \Sigma^{-1} v=\Delta^{2}$ is held fixed. The Bayes rule in $\Phi^{* *}$ against the above prior distribution decides $\theta \in \theta_{1}$ iff

$$
\text { (4.7) } \quad \mathrm{m}_{12}<0
$$

To see this, note that for $a>0$

$(4.8) \quad(a+x)^{j}<(a-x)^{j}$ 
for any positive $j$ if $x<0$. The relation (4.7) is the same as (1.7) for $\lambda=1$. It now follows easily that the rule $\psi_{L}^{1}$ is admissible and minimax in $\bar{\Phi}^{* *}$ [4]. Das Gupta [4] has also shown that $\psi_{L}^{1}$ is the unique (a.e.) minimax in $\bar{\Phi}^{* *}$ if the loss for any correct decision is zero and the loss for deciding $\mu=\mu_{i}$ incorrectly is

$$
\ell\left[\left(1+1 / n_{i}\right)^{-1}\left(\mu-\mu_{i}\right)^{-} \Sigma^{-1}\left(\mu-\mu_{i}\right)\right] \text {, }
$$

where $\ell$ is a positive-valued, bounded, continuous function such that $\ell(\Delta) \rightarrow 0$ as $\Delta+0$.

Again for this case Rao [15] considered the class $\bar{\phi}^{* *}$ of rules whose probabilities of misclassification depend only on $\Delta^{2}$ given in (3.10). Then he derived the optimum rule which minimizes the expression given by (3.11) subject to the condition of similarity for the subset of the parameters given by $\mu_{1}=\mu_{2}$. The optimum rule decides $\mu=\mu_{1}$ iff

$$
\begin{aligned}
& a\left[\left(\mathrm{X}-\overline{\mathrm{X}}_{1}\right)-\left(1+1 / \mathrm{n}_{1}\right)\left(\mathrm{X}-\overline{\mathrm{X}}_{2}\right)\right]^{-} \mathrm{B}^{-1}\left[\left(\mathrm{X}-\overline{\mathrm{X}}_{1}\right)-\left(1+1 / \mathrm{n}_{1}\right)\left(\mathrm{X}-\overline{\mathrm{X}}_{2}\right)\right] \\
& -\mathrm{b}\left[\left(\mathrm{X}-\overline{\mathrm{X}}_{2}\right)-\left(1+1 / \mathrm{n}_{2}\right)\left(\mathrm{X}-\overline{\mathrm{X}}_{1}\right)\right]-\mathrm{B}^{-1}\left[\left(\mathrm{X}-\overline{\mathrm{X}}_{2}\right)-\left(1+1 / \mathrm{n}_{2}\right)\left(\mathrm{X}-\overline{\mathrm{X}}_{1}\right)\right] \\
& \quad \geq c(\mathrm{~B}),
\end{aligned}
$$

where

$$
\text { (4.11) } \quad \begin{aligned}
\mathrm{B}=\mathrm{mS} & +\frac{\mathrm{n}_{1} \mathrm{n}_{2}}{1+\mathrm{n}_{1}+\mathrm{n}_{2}}\left[\left(1+1 / \mathrm{n}_{2}\right)\left(\mathrm{X}-\overline{\mathrm{x}}_{1}\right)^{\prime}\left(\mathrm{x}-\overline{\mathrm{x}}_{1}\right)\right. \\
& \left.+\left(1+1 / \mathrm{n}_{1}\right)\left(\mathrm{X}-\overline{\mathrm{x}}_{2}\right)^{\prime}\left(\mathrm{X}-\overline{\mathrm{x}}_{2}\right)-2\left(\mathrm{X}-\overline{\mathrm{x}}_{1}\right)^{-}\left(\mathrm{X}-\overline{\mathrm{x}}_{2}\right)\right] .
\end{aligned}
$$

It is not clear why Rao imposed the similarity condition even after restricting to the class $\Phi^{*}$. One may directly consider the class of rules invariant under (4.1) and try to minimize (3.11) subject to the condition that $G_{i}(\phi ; 0)$ is equal to a specified constant. Using (4.3) it can be found that the optimum rule decides $\mu \quad \mu_{1}$ iff 


$$
\begin{aligned}
& \text { (4.12) } \quad a\left(k_{1}^{2} m_{11}+k_{2}^{2} m_{22}+\left(k_{1}^{2}+k_{2}^{2}\right)|M|-2 k_{1} k_{2} m_{12}\right)\left(1+1 / n_{2}\right)^{-1} \\
& -b\left(k_{1}^{2} m_{11}+k_{2}^{2} m_{22}+\left(k_{1}^{2}+k_{2}^{2}\right)|M|+2 k_{1} k_{2} m_{12}\right)\left(1+1 / n_{1}\right)^{-1} \\
& >\lambda \operatorname{det}\left(I_{2}+M\right) \text {. }
\end{aligned}
$$

As in (2.29) a similar region for $\theta_{1}$ may be constructed for this case also. It is given by the following:

(4.13) $\mathrm{Y}_{2}^{\prime}\left(\mathrm{mS}+\mathrm{Y}_{1} \mathrm{Y}_{1}^{-}\right)^{-1} \mathrm{Y}_{1} /\left[\mathrm{Y}_{2}^{-}\left(\mathrm{mS}+\mathrm{Y}_{1} \mathrm{Y}_{1}^{\prime}\right)^{-1} \mathrm{Y}_{2}\right]^{\frac{1}{2}}>\mathrm{k}$,

where $Y_{1}$ and $Y_{2}$ are given in (2.15) and (2.16) in vector notations. 
5. Multivariate Case: $\mu_{1}$ and $\mu_{2}$ known

In this case the plug-in rules are given by the following: Decide. $\mu=\mu_{1}$ if

(4.14) $\left(x-\mu_{1}\right) \cdot A^{-1}\left(x-\mu_{1}\right)-\left(x-\mu_{2}\right) \cdot A^{-1}\left(x-\mu_{2}\right)>\lambda$,

where

(4.15) $\quad A=\left[m S+n_{1}\left(\bar{x}_{1}-\mu_{1}\right)\left(\bar{x}_{1}-\mu_{1}\right)+n_{2}\left(\bar{x}_{2}-\mu_{2}\right)\left(\bar{x}_{2}-\mu_{2}\right)\right]$.

On the other hand, a likelihood-ratio rule decides $\mu=\mu_{1}$ iff

$$
\frac{1+\left(x-\mu_{2}\right) \cdot A^{-1}\left(x-\mu_{2}\right)}{1+\left(x-\mu_{1}\right) \cdot A^{-1}\left(x-\mu_{1}\right)}>\lambda(0<\lambda) \text {. }
$$

Define $m^{*}=m+2$

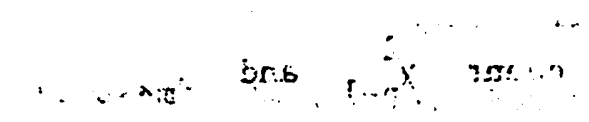

Without loss of generality we may assume that $\mu_{1}=0$ and $\mu_{2}^{\prime}=(1,0, \ldots 0)$. Then the problem is invariant under the following transformations:

$(4.17)(X, A) \rightarrow\left(L X, L A L^{\prime}\right)$,

where $L$ is a nonsingular $p \times p$ matrix of the form

$$
\mathrm{L}=\left[\begin{array}{c|c}
1 & \mathrm{~L}_{12} \\
\hline 0 & \mathrm{~L}_{22}
\end{array}\right]
$$


It can be seen that a set of maximal invariants is given by $\left(\mathrm{x}_{1.2}, \mathrm{x}_{(2)}^{\prime} \mathrm{A}_{22}^{-1} \mathrm{X}_{(2)}, \mathrm{A}_{11.2}\right)$, where

$$
A=\left[\begin{array}{c|c}
A_{11} & A_{12} \\
\hline A_{21} & A_{22} \\
1 & p-1
\end{array}\right]_{p-1}^{1}, x=\left(\begin{array}{c}
x_{1} \\
x_{(2)}
\end{array}\right)_{p-1}^{1}
$$

(4.20) $\quad A_{11.2}=A_{11}-A_{12} A_{22}^{-1} A_{21}$,

(4.21) $\quad \mathrm{x}_{1.2}=\mathrm{x}_{1}-\mathrm{A}_{12} \mathrm{~A}_{22}^{-1} \mathrm{x}_{(2)}$

$\mathrm{A}_{11.2}$ is distributed, independently of $\left(\mathrm{X}_{1.2}, \mathrm{X}_{(2)}^{\prime} \mathrm{A}_{22}^{-1} \mathrm{X}_{12}\right)$, as $\sigma_{11.2} x_{m^{*}-p+1}^{2}$; given $x_{(2)}^{\prime} A_{22}^{-1} X_{(2)}$, the distribution of $x_{1.2}$ is $N\left(d, \sigma_{11.2}\left(1+x_{(2)}^{\prime} \dot{A}_{22}^{-1} X_{(2)}\right)\right)$, and $x_{(2)}^{\prime} A_{22}^{-1} X_{(2)}$ is distributed as the ratio of independent $\chi_{\mathrm{p}-1}^{2}$ and $\dot{\chi}_{\mathrm{m} *-\mathrm{p}+2}^{2}$ variates. In the above $\mathrm{d}$ is equal to 0 or 1 according as $\mu=\mu_{1}$ or $\mu=\mu_{2}$, and $\sigma_{11.2}$ is the residual variance of $\mathrm{x}_{1}$ given $\mathrm{x}_{(2)}$. It can be shown now that the following rule is minimax (and Bayes) in the class of rules invariant under (4.18): Decide $\mu=\mu_{1}$ iff

(4.22) $x_{1.2}<1 / 2$.

The relation (4.22) is the same as (4.14) for $\lambda=0$, and as (4.16) for $\lambda=1$. The above region is not similar for $\mu=\mu_{1}$. Such a simular region may be constructed using

(4.23) $\mathrm{x}_{1.2}\left(1+\mathrm{x}_{(2)}^{\prime} \mathrm{A}_{22}^{-1} \mathrm{X}_{(2)}\right)^{-\frac{1}{2}}\left(\mathrm{~A}_{11.2} /\left(\mathrm{m}^{*}+\mathrm{p}+1\right)\right)^{-\frac{1}{2}}$

which is distributed as Student's' $t$ - distribution with $\mathrm{m}^{*}-\mathrm{p}+1$ degrees of freedom when $\mu=\mu_{1}$. The Mahalanobis distance is equal to $\left(\sigma_{11.2}\right)^{-\frac{1}{2}}$ 
in this case. The probabilities of correct classification for the rule given by (4.22) are the same and they decrease as $p$ increases if $\sigma_{11.2}$ is held fixed.

This section is new in the literature and it is due to the present author. 


\section{$\underline{\text { Referneces }}$}

[1] Anderson, T.W. (1951). Classification by multivariate analysis. Psychometrika $16,31-50$.

[2] Anderson, T.W. (1958). An Introduction to Multivariate Statistical Analysis. Wiley, New York.

[3] Das Gupta S. and Bhattacharya, P.K. (1964). Classification into exponential populations. Sankhya, Ser. A, 26, 17-24.

[4] Das Gupta, S. (1965). Optimum classification rules for classification into two multivariate normal populations. Ann. Math. Statist. 36, 1174-1184.

[5] Das Gupta S. and Kinderman A. (1974). Classifiability and designs for sampling. Sankhya Ser. A, 36, 237-250.

[6] Das Gupta, S. (1974). Probability inequalities and errors in classification. Ann. Statist., 2, 751-762.

[7] Fisher, R.A. (1936). The use of multiple measurements in taxonomic problems. Ann. Eugenics 7, 179-188.

[8] Fisher, R.A. (1938). The statistical utilization of multiple measurements. Ann. Eugenics $8,376-386$.

[9] Kiefer, J. and Schwartz, R. (1956). Admissible Bayes character of $\mathrm{T}^{2}-, \mathrm{R}^{2}-$, and other fully invariant tests for classical multivariate normal problems. Ann. Math. Statist. 36, 747-770.

[10] Kinderman, A. (1972). On some problems in classification. Tech. Rep. 178 , School of Statistics, University of Minnesota, Minneapolis.

[11] Kudo, A. (1959). The classificatory problem viewed as a two-decision problem. Memoirs of Faculty of Science Kuyushu University, Ser. A, $13,96-125$.

[12] Schaafsma, W. (1971). Testing statistical hypotheses concerning the expectations of two independent normals; both with variance 1 . I and II. Proc. Kon. Ned. Ak. (Indag. Math.) 33, 86-105.

[13] Schaafsma, W. and Van Verk, G.N. (1977). Classification and discrimination problems with applications. Statistica Neerlandica, $31,25-45$.

[14] Sitgreaves, R. (1952). On the distribution of two random matrices used in classification procedures. Ann. Math. Statist. 23, 263-270. 
[15] Rao, C.R. (1954). A general theory of discrimination when the information about alternative population distributions is based on samples. Ann. Math. Statist., 25, 651-670.

[16] Von Mises, R. (1945). On the classification of observation data into distinct groups. Ann. Math. Statist., 16, 68-73.

[17] Wald, A. (1944). On a statistical problem arising in the classification of an individual into one of two groups. Arn. Math. Statist., $15,145-162$. 